Article

\title{
Dynamic of Lachancea thermotolerans Population in Monoculture and Mixed Fermentations: Impact on Wine Characteristics
}

\author{
Pilar Blanco *, Eva Rabuñal, Noemi Neira and David Castrillo \\ Estación de Viticultura e Enoloxía de Galicia (EVEGA-AGACAL), Ponte San Clodio S/N, 32428 Leiro, Ourense, \\ Spain; eva.bio.256@gmail.com (E.R.); neira.022@gmail.com (N.N.); david.castrillo.cachon@xunta.gal (D.C.) \\ * Correspondence: pilar.blanco.camba@xunta.gal; Tel.: +34-988-78-80-91
}

Received: 28 March 2020; Accepted: 18 May 2020; Published: 1 June 2020

check for updates

\begin{abstract}
Lachancea thermotolerans is a non-Saccharomyces yeast appreciated for its potential of acidification due to the production of lactic acid; however, this species also synthetizes other metabolites that modulate organoleptic wine properties. The aim of this study was to evaluate the strain L. thermotolerans Lt93 to ferment 'Treixadura' and 'Mencía' musts and its impact on yeast population dynamics and wine characteristics. Fermentations using monocultures of L. thermotolerans Lt93 and S. cerevisiae strains, sequential inoculation and spontaneous process were performed. The dynamic of yeast population and wine composition were analyzed following standard methodology. L. thermotolerans Lt93 was unable to overgrow wild yeast population in 'Treixadura' white must; however, with 'Mencía' red must, Lt93 was the predominant yeast at the beginning of fermentation and remained at high frequency until the end. Lt93 Treixadura wines had slightly higher acidity and higher content of esters and acids than ScXG3 wines. Lt93 Mencía wines presented higher acidity $(10.1 \mathrm{~g} / \mathrm{L})$ and $0.8 \%(v / v)$ lower ethanol content than Sc71B wines. The content of esters and fatty acids was 3.3 and 4.0 times lower, respectively, in Lt93 than in Sc71B Mencía wines. It was possible to increase wine acidity and modulate the chemical wine profile by using Lt93.
\end{abstract}

Keywords: non-Saccharomyces; Lachancea thermotolerans; Saccharomyces cerevisiae; monoculture fermentation; sequential fermentation; wine

\section{Introduction}

The fermentation of grape juice into wine is a complex microbiological and biochemical process in which yeast, responsible for alcoholic fermentation, plays an essential role. Not only does yeast transform grape sugars into ethanol and carbon dioxide, they also produce a large number of secondary compounds. These metabolites include higher alcohols, volatile acids, esters, carbonyl compounds, volatile phenols, sulfur-containing compounds and thiols; although minor, they are very important from a sensorial point of view $[1,2]$.

Spontaneous fermentations involve a sequential succession of yeasts: during early stages, several yeast species of genera Hanseniaspora, Candida, Pichia and Metschnikowia are present; however, as fermentation progresses, these non-Saccharomyces species are naturally substituted by Saccharomyces because the latter is a good fermenter and has high alcohol tolerance [3,4]. Traditionally, the presence of non-Saccharomyces yeast was associated to undesirable compounds and they were considered spoilage microorganisms [5]. However, nowadays their benefits on wine quality are widely recognized [6]. Non-Saccharomyces yeast synthetize metabolites that contribute to wine quality; moreover, they also produce enzymes to optimize certain steps during winemaking [7]. Later on, their role in alcohol reduction and acidification of wines add an extra value to these yeasts, which can be used 
as a tool to mitigate the consequences of climate change in wine [8]. In this context, the potential of non-Saccharomyces yeast in winemaking has been re-evaluated [6]; thus, nowadays, there are several strains of non-Saccharomyces yeasts commercially available as active dry products [9].

Non-Saccharomyces yeasts have limited fermentation power and they are not able to dominate the wild yeast populations in must; however, their activity in early stages of fermentation is enough to contribute positive changes in wine quality. Therefore, to take advantage of their desirable oenological traits, it is necessary to inoculate a strain of $S$. cerevisiae in order to complete fermentation. Thus, the use of controlled mixed fermentations has been proposed $[4,10]$. Numerous studies have reported the use of non-Saccharomyces as monoculture and in mixed fermentation (simultaneous or sequential) compared to $S$. cerevisiae; most of them conclude that sequential inoculation is usually the best option $[5,11,12]$.

Among non-Saccharomyces yeast, Lachancea thermotolerans (formerly Kluyveromyces thermotolerans) is widely used at industrial level to acidify wines, especially in warm viticultural regions $[9,13]$. Indeed, the results of numerous studies about the oenological properties of L. thermotolerans confirmed its suitability to obtain quality wines [14]. The first reports about L. thermotolerans highlighted the high production of lactic acid during alcoholic fermentation; consequently, they also evidenced the acidification ability of this yeast [15-17]. Further studies corroborate these findings (for a review see [9]). Moreover, L. thermotolerans used in combination with $S$. cerevisiae reduced the ethanol content of wines $0.7-0.9 \%(v / v)$ [11] or from $0.2 \%(v / v)$ to $0.35 \%(v / v)$ [18-20]. This fact, along with its potential to increased total wine acidity, make the application of L. thermotolerans a suitable strategy to face climate change consequences in oenology $[8,21]$. Additional effects of this yeast include a reduction of $0.46 \mathrm{~g} / \mathrm{L}$ in volatile acidity [22] and the consumption of acetic acid [23]; an increase of $2.2 \mathrm{~g} / \mathrm{L}$ of glycerol content in industrial fermentations [11]; significant variations in the content of higher alcohols, esters or terpenic compounds [5,12,21,24]; or an increase from 1.3- to 2.0-fold of 2-phenylethanol [5,11]. All these characteristics are involved in wine complexity resulting in wines differentiated at sensory level [11,12]. However, it is important to mention that some of these metabolic properties of L. thermotolerans are strain-dependent $[14,19,22,25]$; therefore, more research is necessary about the isolation and selection of L. thermotolerans strains using specific criteria for their future exploitation [9].

Recent studies have revealed the existence of genetic variation in the microbial communities from different viticultural areas [26]. Such diversity resulted in phenotypic differences responsible for the regional signatures in wines $[27,28]$. Accordingly, the oenological performance of L. thermotolerans strains was influenced by their geographical and ecological origin [14,29]. Moreover, the use of mixed cultures could be used to enhance the varietal expression of certain cultivars [12].

In Galicia (NW of Spain) the wine industry constitutes an important sector from both social and economic points of view. A wide climatic and orographic heterogeneity, together with the growth of different traditional grapevine varieties reinforce the concept of terroir in this region. Among the Vitis vinifera L. cultivars grown in Galicia, 'Treixadura' and 'Mencía' are two of the most representative ones. Moreover, recent studies evidenced the existence of a biogeographic pattern in the yeast population that could be associated to a particular microbial terroir and wine typicality in certain areas of Galicia [30]. The results associated the presence of L. thermotolerans to Treixadura and Mencía musts in Denomination of Origin (DO) Ribeiro and DO Monterrei. Preliminary studies at laboratory scale on the oenological potential of non-Saccharomyces yeasts highlighted the strain L. thermotolerans Lt93 by its potential to acidify wine and modulate its sensory profile [31] positively. However, the results obtained with this strain at winery scale by sequential fermentations indicated that further research was necessary [32].

The purpose of this work was to evaluate the potential of L. thermotolerans Lt93 to ferment Treixadura and Mencía musts under different inoculation modalities as well as its impact on fermentation kinetics, yeast population dynamics and wine characteristics. The results showed the advantage of using the strain Lt93 to acidify wine and modulate its volatile composition and sensory profile. 


\section{Materials and Methods}

\subsection{Grapevine Cultivars and Yeast Strains}

Fermentations were carried out using must of two traditional Vitis vinifera L. cultivars from Galicia (NW Spain): 'Treixadura' and 'Mencía.' 'Treixadura' is a white cultivar grown in all Galician Denominations of Origin (DO) with a relevant role in DO Ribeiro [33]. The culture of the red variety 'Mencía' is also widespread in Galicia and close regions [34], but is especially important in DO Ribeira Sacra.

Saccharomyces cerevisiae ScXG3 and Lachancea thermotolerans Lt93 are authocthonous strains from the yeasts culture collection maintained at Estación de Viticultura e Enoloxía de Galicia (EVEGA). S. cerevisiae ScXG3 is a strain that has shown desirable fermentation abilities at pilot scale and it enhances wine quality $[35,36]$. L. thermotolerans Lt93 has been selected after preliminary studies at laboratory scale [31]. S. cerevisiae Lalvin $71 \mathrm{~B}^{\circledR}$ (Lallemand) is a commercial strain isolated in Narbonne, France. Lalvin $71 \mathrm{~B}^{\circledR}$ is a high ester producer and it has low nitrogen demand; in addition, it can undertake malo-etanolic fermentation.

\subsection{Yeast Growth and Inocula Preparation}

Yeast strains were grown in YPD (yeast extract $1 \% w / v$, peptone $2 \% w / v$, glucose $2 \% w / v$, and agar $2 \% w / v$ for solid media) at $28{ }^{\circ} \mathrm{C}$ and stored at $4{ }^{\circ} \mathrm{C}$. Inocula for fermentation assays were obtained as follows: a pre-inoculum of each strain was grown in liquid YPD overnight at $28^{\circ} \mathrm{C}$. Then, it was transferred to a $2 \mathrm{~L}$ Erlenmeyer flask containing $1 \mathrm{~L}$ of YPD and incubated at $28{ }^{\circ} \mathrm{C}, 150 \mathrm{rpm} \mathrm{for} 24 \mathrm{~h}$ in a SANYO orbital incubator. The cells were recovered by centrifugation at $7025 \times g$ for $10 \mathrm{~min}$ in an Avanti ${ }^{\circledR}$ J-26 XP centrifuge (BECKMAN COULTER, Madrid, Spain), washed with sterile water and re-suspended in $100 \mathrm{~mL}$ of saline solution. The inocula concentration was calculated by serial dilutions and spread on YPD plates in duplicate. Plates were incubated at $28{ }^{\circ} \mathrm{C}$ and those containing between 20 and 200 colonies were used for viable cells counting.

\subsection{Vinifications}

Organic must from 'Treixadura' was kindly provided by Cume do Avia winery (Ourense, Spain) in 2018 vintage. Its chemical characteristics are summarized in Table 1 . This must was distributed in $1 \mathrm{~L}$ bottles and they were inoculated (in triplicate) with $1 \times 10^{8}$ cells/mL of the following yeasts:

1. S. cerevisiae ScXG3

2. L. thermotolerans Lt93

3. L. thermotolerans Lt93 + S. cerevisiae ScXG3 by sequential fermentation (Lt93 + ScXG3)

4. No yeast addition (spontaneous fermentation with indigenous yeast from the must) (Spo)

Table 1. Characteristics of Treixadura and Mencía musts used in this study.

\begin{tabular}{ccc}
\hline Parameter & Treixadura & Mencía \\
\hline${ }^{\circ}$ Brix & 22.4 & 21.5 \\
Sugars $(\mathrm{g} / \mathrm{L})$ & 219.5 & 209.0 \\
Total acidity $(\mathrm{g}$ tartaric acid/L) & 5.7 & 4.6 \\
$\mathrm{pH}$ & 3.51 & 3.57 \\
Malic acid $(\mathrm{g} / \mathrm{L})$ & 2.3 & 1.4 \\
Tartaric acid $(\mathrm{g} / \mathrm{L})$ & 4.7 & 4.8 \\
\hline
\end{tabular}

Fermentations were carried out at $18{ }^{\circ} \mathrm{C}$. For sequential fermentation, Lt93 was inoculated first; then, after the fermentation had begun and the ${ }^{\circ}$ Brix had decreased about 5 points (day 4 ), the second strain (ScXG3) was added. 
Grapes from 'Mencía' were harvested manually during the 2019 vintage in vineyard EVEGA and transported to the experimental winery of EVEGA. At the winery, the grapes were de-stemmed, crushed and pressed in a hydraulic press to separate must and marc. During grape processing, $50 \mathrm{mg} / \mathrm{L}$ of $\mathrm{SO}_{2}$ were added to prevent oxidation as well as for microbiological control. Then, they were homogeneously distributed in $5 \mathrm{~L}$ stainless steel tanks ( $2.8 \mathrm{~L}$ of must $+1.7 \mathrm{~kg}$ of marc in each tank). Must characteristics are summarized in Table 1 . The mass was inoculated with $2 \times 10^{7}$ cells $/ \mathrm{mL}$ of yeast inoculum and fermented in duplicate at room temperature. The following yeast strains were used:

1. S. cerevisiae Sc71B

2. L. thermotolerans Lt93

3. L. thermotolerans Lt93 + S. cerevisiae Sc71B by sequential inoculation (Lt93 + Sc71B)

4. No yeast addition (spontaneous fermentation with indigenous yeast from the must) (Spo)

S. cerevisiae Sc71B was added as recommended by the manufacturer: $20 \mathrm{~g} / \mathrm{hL}$ of yeast were rehydrated in warm water $\left(35-40^{\circ} \mathrm{C}\right)$ for $20 \mathrm{~min}$ by gently stirring, acclimated gradually by mixing with grape juice and inoculated into the must. Fermentations were performed at room temperature. For sequential fermentation, Lt93 was inoculated first; then, after the fermentation had started and the ${ }^{\circ}$ Brix had decreased about 5 points (day 2), the second strain (Sc71B) was added. After five days of maceration, the fermentations were manually pressed and allowed to finish.

The development of alcoholic fermentation was monitored by daily measurement of ${ }^{\circ}$ Brix (using a digital refractometer) and temperature. In addition, samples were taken from the must and at the beginning $(\mathrm{Fi})$, tumultuous $(\mathrm{Ft})$ and final $(\mathrm{Ff})$ stages of fermentation for microbiological control. When the fermentations ended, white wines were centrifuged, sulfited $\left(25 \mathrm{mg} / \mathrm{L}\right.$ of free $\left.\mathrm{SO}_{2}\right)$ and stored until further chemical and sensory analysis. Red wines were racket and kept at room temperature to allow spontaneous malolactic fermentation; when malic acid content was not detected (after 14 days in S. cerevisiae Sc71B fermentations and 40 days in spontaneous ones), the wines were racked again and sulfited $\left(25 \mathrm{mg} / \mathrm{L}\right.$ of free $\left.\mathrm{SO}_{2}\right)$. Finally, red wines were bottled and stored until further chemical analysis.

\subsection{Microbiological Control}

Must samples and those taken at different stages of fermentation were used to determine yeast population dynamic during the vinification process. The samples were serial diluted in $2 \% w / v$ buffered peptone water and spread on WL Nutrient Agar medium (Scharlau Microbiology, Barcelona, Spain) [37]. Plates were incubated at $28^{\circ} \mathrm{C}$ until visible colonies appeared; those containing between 20 and 200 colonies were used to quantify the total viable cells and the frequency of each type of yeast based on their colony morphotype. Then, a representative number of colonies based on their aspect and frequency (10-20 for each sample) were selected randomly and isolated on YPD for further characterization. Saccharomyces and non-Saccharomyces yeasts were distinguished by growth on Lysine medium (Thermo Scientific ${ }^{\mathrm{TM}}$ Oxoid $^{\mathrm{TM}}$, Madrid, Spain), since the former ones are unable to grow on this medium.

Yeast species identity was confirmed by PCR amplification of the 5.8S rRNA gene and the two internal (non-coding) ITS1 and ITS2 spacers using the ITS1 and ITS4 primers [38]. S. cerevisiae isolates were characterized at the strain level by analysis of mitochondrial DNA restriction profiles (mtDNA-RFLPs). Total yeast DNA was obtained as described by Querol [39] and digested with the restriction endonuclease Fast digest HinfI (ThermoFisher Scientific, Madrid, Spain). The restriction fragments were separated by gel electrophoresis on a $0.8 \%(w / v)$ agarose gel in 1X TBE (ThermoFisher Scientific, Madrid, Spain). After having stained with ethidium bromide $(0.5 \mu \mathrm{g} / \mathrm{mL})$, the DNA pattern bands were visualized under UV light and documented using a Molecular Imager ${ }^{\circledR}$ Gel Doc $^{\mathrm{TM}} \mathrm{XR}+$ imaging system (BIO-RAD, Madrid, Spain). 


\subsection{Chemical Analysis}

Must parameters, including ${ }^{\circ}$ Brix, sugar content, total acidity, $\mathrm{pH}$, malic acid and tartaric acid, were determined using the official methodology [40]. Basic parameters of wines (alcohol content, reducing sugars, $\mathrm{pH}$, titratable and volatile acidity, tartaric, malic and lactic acids) were determined by Fourier transform infrared spectrometry (FTIR) using a Wine Scan FT120 analyzer (FOSS Electric, Barcelona, Spain) calibrated according to OIV [40]. In addition, the free and total sulfur dioxides were also quantified using the OIV methods.

Wine volatile compounds were quantified by gas chromatography (GC) [33]. Major volatile compounds (acetaldehyde, ethyl acetate, methanol and higher alcohols) were quantified by direct injection using a 7890A gas chromatograph with flame ionization detector (FID) (Agilent Technologies, Palo Alto, CA, USA). Minor volatile compounds (volatile fatty acids, ethyl esters of fatty acids and acetates of higher alcohols) were extracted (solid phase extraction) according to Bouzas-Cid [33]. After that, the extract was injected in an Agilent 6890 (Agilent Technologies, Palo Alto, CA, USA) gas chromatograph coupled to an Agilent 5973N selective mass spectrometer (MS) detector with a single quadrupole analyzer.

The compounds were identified by comparison of their mass spectra and retention times to those of the pure standards using the National Institute of Standards andTechnology (NIST) Mass Spectral Library. For quantification, the peak area of each compound was calculated by integration and compared to an internal standard of known concentration. All determinations were carried out in duplicate. In addition, the odor activity value (OAV) for each compound was calculated as the ratio between its concentration and its perception threshold [2,41-46]; those compounds with an OAV > 1 are considered to contribute to wine aroma.

\subsection{Sensory Evaluation}

White wines were evaluated at sensory level by a tasting panel with experience in Galician wines. The tasting sessions were held in November at the tasting room of EVEGA. The panel consisted of eight judges, six males and two females aged between 35 and 60 years old. A descriptive score card including 21 descriptors and global wine quality was used [32]. The selected descriptors were specifically chosen for Galician white wines and were scored from 0 (absent) to 9 (most intense). Wine samples (30 mL) were coded and presented arbitrarily to the panel in clear tulip-shaped glasses.

\subsection{Statistical Analysis}

The differences in chemical composition of wines, considering the yeast strain as a factor, were checked by one-way ANOVA. A Tukey HSD test was used to separate means. These analyses were carried out using SPSS18.0 for Windows.

Sensory descriptors were classified for each wine by comparing the percentage of the geometric means (GM\%). GM was calculated as square root of the product between relative intensity (I\%) and relative frequency (F\%) [47]. Principal component analysis (PCA) was used to separate the wines according to their volatile composition considering those compounds with OAV $>1$ in at least two wines. Previously, data were standardized using the function $f=\frac{(x-\text { mean })}{\text { standard deviation }}$ to guarantee their equity in those variables or factors with different values in different units. The PCA was performed using PAST Version 3.26 (2019).

\section{Results}

\subsection{Fermentation Kinetics and Dinamic of Yeast Population}

\subsubsection{Treixadura}

Figure 1 represents the evolution of ${ }^{\circ}$ Brix and yeast population dynamics during Treixadura fermentations. ScXG3 started fermentation within the first $24 \mathrm{~h}$ with a rapid decrease of ${ }^{\circ}$ Brix until 
day 8 , but the fermentation speed slowed down at the end of the process. Spontaneous fermentations and those inoculated with L. thermotolerans Lt93 showed a similar curve: they began to ferment 2 days later, after which the fermentation speed was as fast as the $S$. cerevisiae one. This fact suggested that Lt93 was unable to overgrow wild yeast population and to begin fermentation.

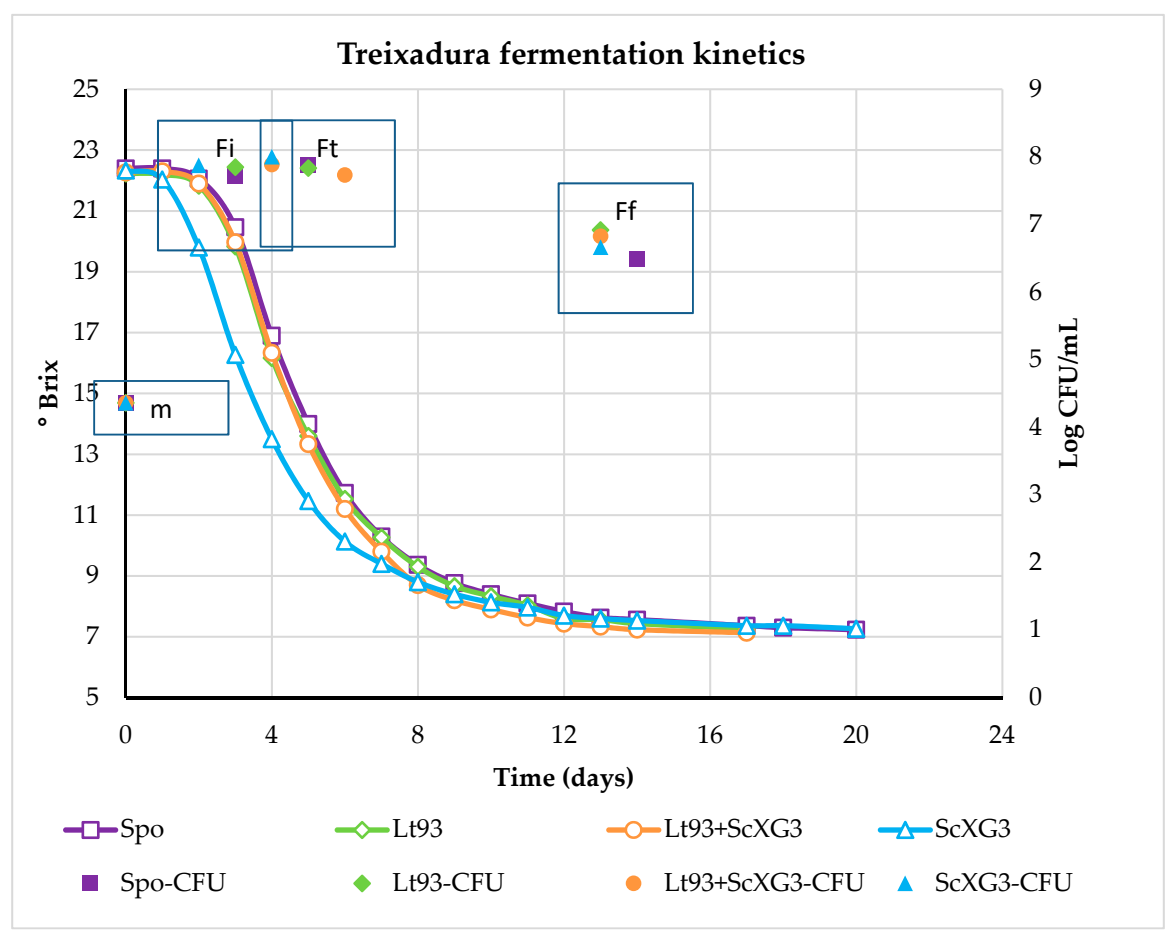

Figure 1. Evolution of ${ }^{\circ}$ Brix and yeast population $(\mathrm{CFU} / \mathrm{mL})$ during fermentation of Treixadura with S. cerevisiae ScXG3 and L. thermotolerans Lt93 in monoculture, sequential fermentation (Lt93+ScXG3) and spontaneous process (Spo). M-must; Fi-initial fermentation; Ft-tumultuous fermentation; Ff-final fermentation.

Regarding quantitative level, Treixadura must contained $4.4 \log$ of colony forming units (CFU)/mL. Yeast population dynamic during fermentation showed similar tendency in ScXG3 and spontaneous processes: their number increased up to $8.0 \log \mathrm{CFU} / \mathrm{mL}$ in tumultuous stage and then decreased at the end (6.5 $\log \mathrm{CFU} / \mathrm{mL})$, as expected. However, in those fermentations with Lt93 the maximum amount of yeast was achieved at the initial stages, decreased slightly at the middle stages, but it remained at values of $6.6 \log \mathrm{CFU} / \mathrm{mL}$ at the end (Figure 1).

Concerning the qualitative level, the yeast species found in Treixadura must were: Candida spp. $(30 \%)$, Saccharomyces cerevisiae (23\%), Pichia anomala (20\%), Hanseniaspora uvarum (17\%), Metschnikowia spp. $(7 \%)$ and L. thermotolerans (3\%). After inoculation of the fermentative species, the implantation ability of S. cerevisiae ScXG3 and L. thermotolerans Lt93 was different (Figure 2). Genetic analysis of isolates from ScXG3 fermentations showed that ScXG3 was the dominant strain at all fermentation stages. However, L. thermotolerans Lt93 was unable to overgrow must yeast population and to control fermentations. Its frequency decreased as fermentation progressed when it was added at monoculture. In this case, six different strains of $S$. cerevisiae were identified, two of them (ScXG3 and ScR4) being co-dominants in the process. Population dynamics in sequential fermentations Lt93+ScXG3 was similar to the previous one. When ScXG3 was added, this fermentation was already spontaneously dominated by ScR4. Spontaneous processes were also controlled by several strains of S. cerevisiae. A total of 13 different strains were identified; however, only two of them (ScXG3 and ScR4) appeared at frequencies higher than $10 \%$, and were thus the main strains responsible for fermentation. 


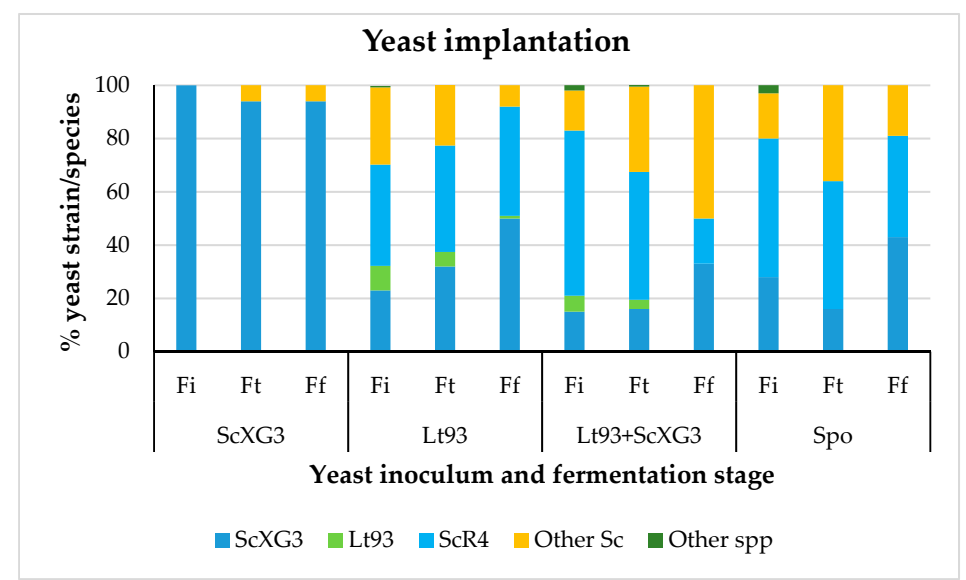

Figure 2. Inoculum implantation in Treixadura fermentations: frequency of each inoculated yeast strain at initial (Fi), tumultuous $(\mathrm{Ft})$ and final (Ff) stage of fermentation. Sc-S. cerevisiae; spp.-species.

\subsubsection{Mencía}

The results of Mencía fermentations were completely different from those observed with Treixadura. Both yeast species used as starters, S. cerevisiae Sc71B and L. thermotolerans Lt93, started fermentation within the first two days; however, in Lt93, the ${ }^{\circ}$ Brix decrease slowed down close to the end (Figure 3). Spontaneous fermentation started after three days but its speed was similar to the commercial S. cerevisiae. Yeast population in Mencía must was $6.02 \mathrm{log}$ CFU/mL. The amount of yeasts increased until $7.9 \log \mathrm{CFU} / \mathrm{mL}$ at the beginning and middle stages in fermentations with $S$. cerevisiae and decreased at the end. L. thermotolerans fermentations showed lower yeast counts than $S$. cerevisiae trials at all stages: they reached up to $7.5 \log \mathrm{CFU} / \mathrm{mL}$ in Fi and $\mathrm{Ft}$ and decreased at final stages until $6.2 \log$ $\mathrm{CFU} / \mathrm{mL}$.

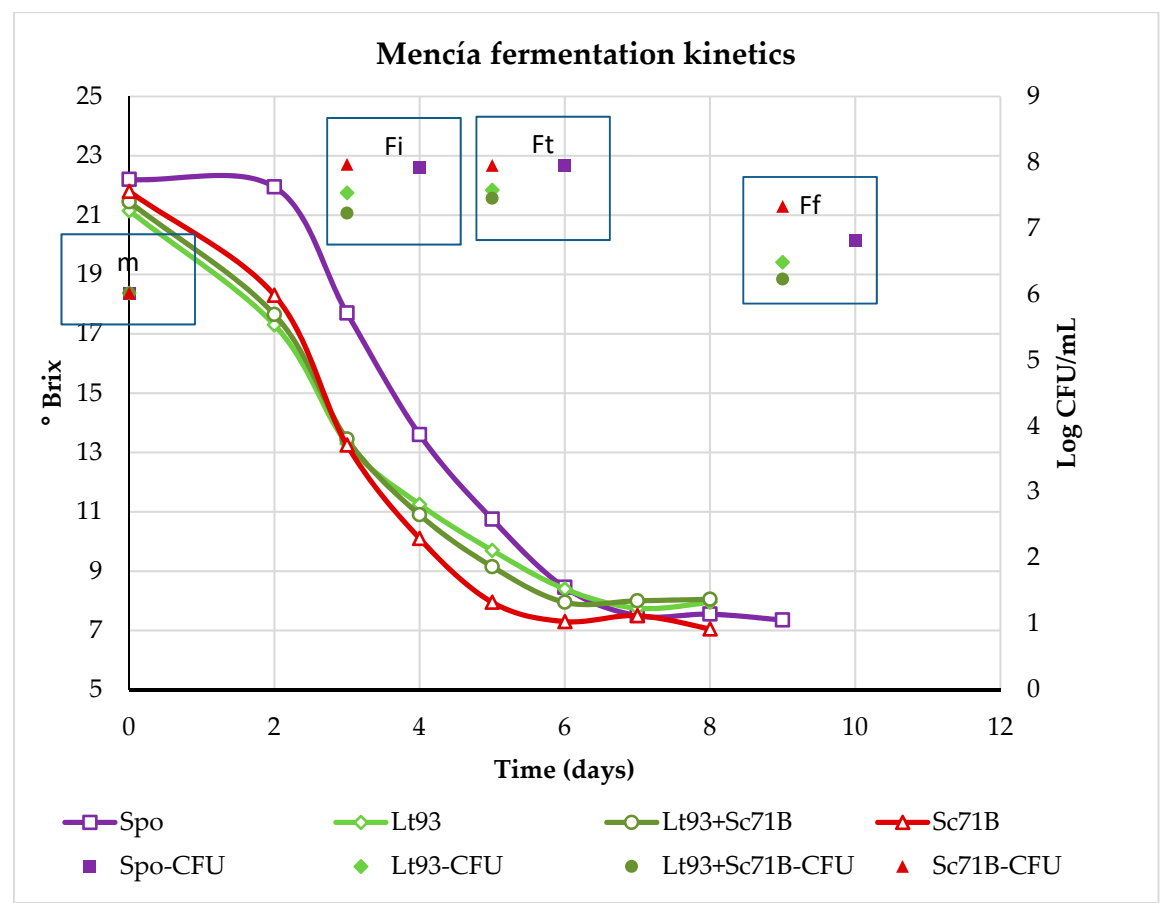

Figure 3. Evolution of ${ }^{\circ}$ Brix and yeast population $(\mathrm{CFU} / \mathrm{mL})$ during Mencía fermentations with S. cerevisiae Sc71B and L. thermotolerans Lt93 in monoculture, sequential fermentation (Lt93+Sc71B), and spontaneous process (Spo). $\mathrm{m}$-must; $\mathrm{Fi}$-initial fermentation; $\mathrm{Ft}$-tumultuous fermentation; $\mathrm{Ff}$-final fermentation. 
At qualitative level Mencía must contained: H. uvarum (77\%), Metschnikowia spp. (19\%), Pichia kluyveri $(2 \%)$ and Starmerella bacillaris (2\%). As observed in Treixadura, S. cerevisiae strain (Sc71B in this case) showed a good implantation ability when it was added as single inoculum to must. This strain represented $85 \%$ of yeasts at the beginning of fermentation and achieved $100 \%$ at the end (Figure 4). In contrast to Treixadura fermentations, L. thermotolerans Lt93 (added as monoculture) was able to ferment Mencía must and successfully overgrow must microbiota being the dominant yeast $(>90 \%)$ at the beginning and middle of the fermentation. Furthermore, Lt93 remained in high frequency in the final stages of fermentation but in codominance with S. cerevisiae, mainly strain Sc71B (Figure 4). In sequential trials Lt93 was also the dominant yeast at the beginning (>90\%). After S. cerevisiae Sc71B inoculation, Lt93 was replaced gradually as the fermentation progressed, decreasing up to $28 \%$ at the final stages of fermentation. In this case, the sequential inoculum Sc71B was the dominant strain within S. cerevisiae population, reaching frequencies of $78 \%$ and $90 \%$ in tumultuous and final stages, respectively. S. cerevisiae Sc71B appeared also in codominance with ScXXII and other minor S. cerevisiae strains $(n=8)$ in the spontaneous processes (Figure 4).

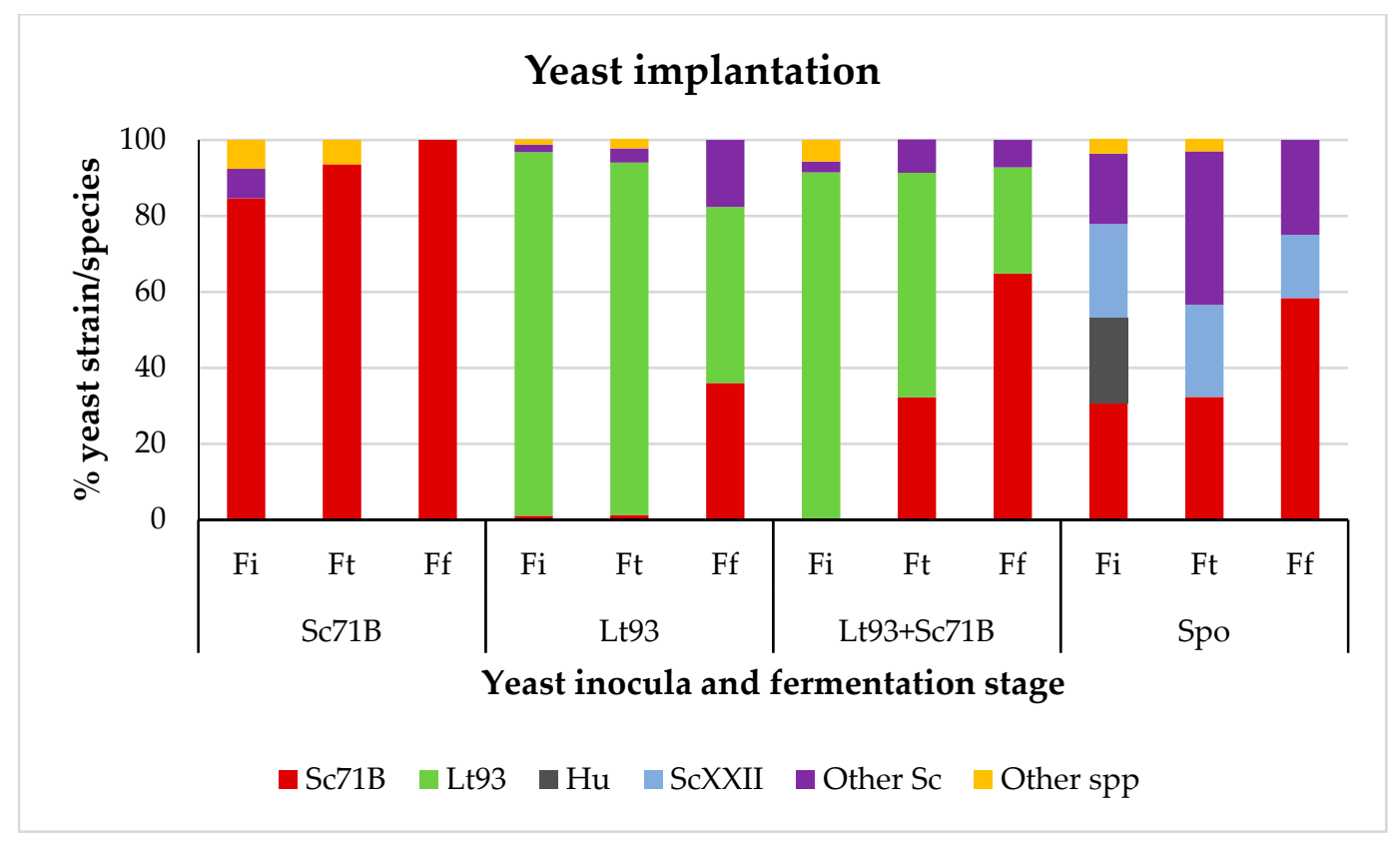

Figure 4. Inoculum implantation in Mencía fermentations: frequency of each inoculated yeast strain at initial (Fi), tumultuous (Ft) and final fermentation (Ff); Hu-Hanseniaspora uvarum; Sc-S. cerevisiae; spp.-species.

\subsection{Chemical Characteristics of Wines}

\subsubsection{Treixadura}

The general composition of Treixadura wines is summarized in Table 2. The results showed significant differences among wines in total acidity, lactic acid, glycerol content and $\mathrm{pH}$. Wines obtained with Lt93 presented higher total acidity values probably due to a higher content of both lactic and tartaric acid. Consequently, they also had lower $\mathrm{pH}$. The remaining parameters were similar for all wines. In addition, those wines involving $\mathrm{Lt} 93$ activity achieved higher values of total $\mathrm{SO}_{2}$. The content of residual sugar after fermentation was $>2.0 \mathrm{~g} / \mathrm{L}$ in three of the wines, being ScXG3 wine the one with the highest amount. 
Table 2. Characteristics of Treixadura wines elaborated with S. cerevisiae ScXG3, L. thermotolerans Lt93, sequential fermentation of both strains and by spontaneous fermentation (Spo).

\begin{tabular}{ccccc}
\hline Chemical Parameter & ScXG3 & Lt93 & Lt93+ScXG3 & Spo \\
\hline Alcohol content (\%vol) & $13.5 \pm 0.2$ & $13.5 \pm 0.1$ & $13.6 \pm 0.1$ & $13.6 \pm 0.0$ \\
Glucose + fructose (g/L) & $4.2 \pm 2.3$ & $3.5 \pm 0.7$ & $1.6 \pm 1.1$ & $2.9 \pm 0.3$ \\
Total acidity (g tartaric acid/L) & $5.6 \pm 0.1^{\mathrm{a}}$ & $5.8 \pm 0.1^{\mathrm{b}}$ & $5.7 \pm 0.1^{\mathrm{ab}}$ & $5.6 \pm 0.0^{\mathrm{a}}$ \\
Volatile acidity (g acetic acid/L) & $0.45 \pm 0.04$ & $0.36 \pm 0.05$ & $0.39 \pm 0.01$ & $0.38 \pm 0.02$ \\
Lactic acid (g/L) & $0.1 \pm 0.0^{\mathrm{a}}$ & $0.2 \pm 0.0^{\mathrm{b}}$ & $0.2 \pm 0.1^{\mathrm{ab}}$ & $0.1 \pm 0.0^{\mathrm{a}}$ \\
Malic acid (g/L) & $1.8 \pm 0.0$ & $1.7 \pm 0.1$ & $1.7 \pm 0.1$ & $1.8 \pm 0.0$ \\
Tartaric acid (g/L) & $3.4 \pm 0.0$ & $3.7 \pm 0.0$ & $3.4 \pm 0.2$ & $3.4 \pm 0.1$ \\
pH (-) & $3.45 \pm 0.02^{\mathrm{a}}$ & $3.39 \pm 0.02^{\mathrm{b}}$ & $3.42 \pm 0.03^{\mathrm{ab}}$ & $3.46 \pm 0.02^{\mathrm{a}}$ \\
Glycerol (g/L) & $3.9 \pm 0.1^{\mathrm{a}}$ & $3.3 \pm 0.1^{\mathrm{b}}$ & $3.5 \pm 0.1^{\mathrm{b}}$ & $3.6 \pm 0.1^{\mathrm{ab}}$ \\
Total sulfur dioxide (mg/L) & $36.7 \pm 3.0^{\mathrm{a}}$ & $54.3 \pm 5.5^{\mathrm{b}}$ & $49.7 \pm 1.1^{\mathrm{b}}$ & $37.3 \pm 2.1^{\mathrm{a}}$ \\
\hline
\end{tabular}

Data are mean values of three replicate fermentations \pm SD. * significant differences according to Tukey's test $(p<0.05){ }^{a, b}$ in the same row indicate significant differences among wines.

Some fermentative volatile compounds were also determined in order to evaluate the effect of the yeast strains on wine aroma profile. Table 3 shows the concentration of 20 compounds including higher alcohols, acetaldehyde, esters and fatty acids. It is worth mentioning that 15 of them achieved concentrations above their odor threshold (Table S1 in Supplementary Materials). Data analysis also showed significant differences among wines in $55 \%$ of these compounds at $p<0.05$. The highest content of higher alcohols was achieved in ScXG3 wines whereas Lt93 wines presented the lowest values mainly due to 2-methyl-1-propanol and 3-methyl-1-butanol content. However, Lt93 wines presented higher amounts of ethyl esters and C6-C12 fatty acids than the remaining wines (Table 3). Regarding C4-C5 fatty acids, their concentration was higher in ScXG3 wines. The wines from spontaneous fermentation (Spo) are highlighted for their content of 2-phenylethyl acetate. It is also important to note the higher content of acetaldehyde in Spo and Lt93+ScXG wines, although the differences were not statistically significant.

Principal component analysis allowed the separation of Treixadura wines based on their volatile composition (Figure 5). The first two components, PC1 and PC2, explained $71.33 \%$ of the variance. ScXG3 wines were plotted on the negative side of PC1 and PC2 characterized by higher concentrations of higher alcohols and $\mathrm{C} 4-\mathrm{C} 5$ fatty acids. Wines from spontaneous fermentations were close to the plot center. In contrast, Lt93 wines were located in the positive side of PC1 and negative side of PC2 due to their higher contents of ethyl esters and $\mathrm{C} 6-\mathrm{C} 12$ fatty acids. However, wine from sequential fermentations with Lt93+ScXG3 appeared close to the Spo wines, as expected. Both wines did not stand out by any particular compound and they were fermented by several S. cerevisiae strains.

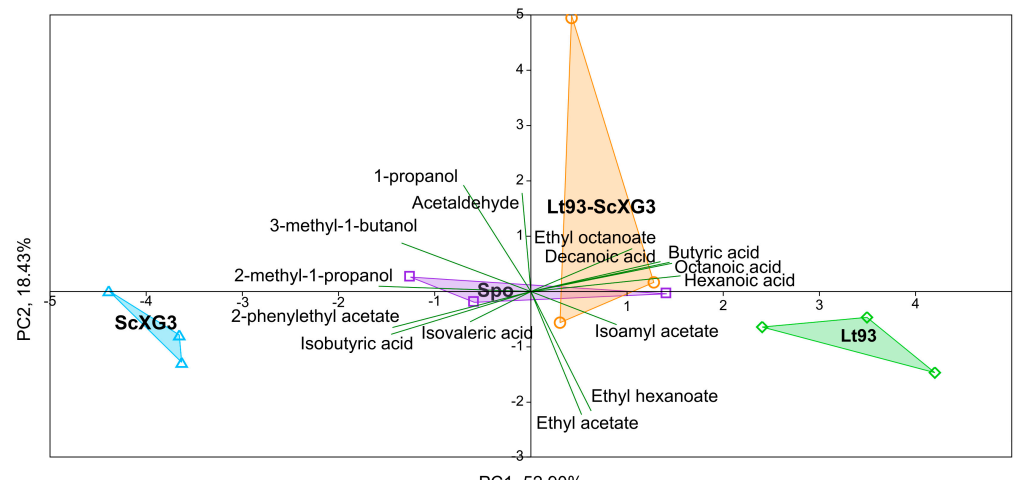

Figure 5. Principal component analysis (PCA) of Treixadura wines: biplot for the first two components (PC) for volatile compounds with $\mathrm{OAV}>1$. Wines were from fermentation with the following yeasts: ScXG3-S. cerevisiae ScXG3; Lt93-L. thermotolerans Lt93; Lt93-ScXG3-L. thermotolerans Lt93 and sequential inoculation of $S$. cerevisiae ScXG3; Spo: spontaneous fermentation. 
Table 3. Concentration of volatile compounds in Treixadura wines elaborated with S. cerevisiae ScXG3, L. thermotolerans Lt93, sequential fermentation of both strains and by spontaneous fermentation (Spo).

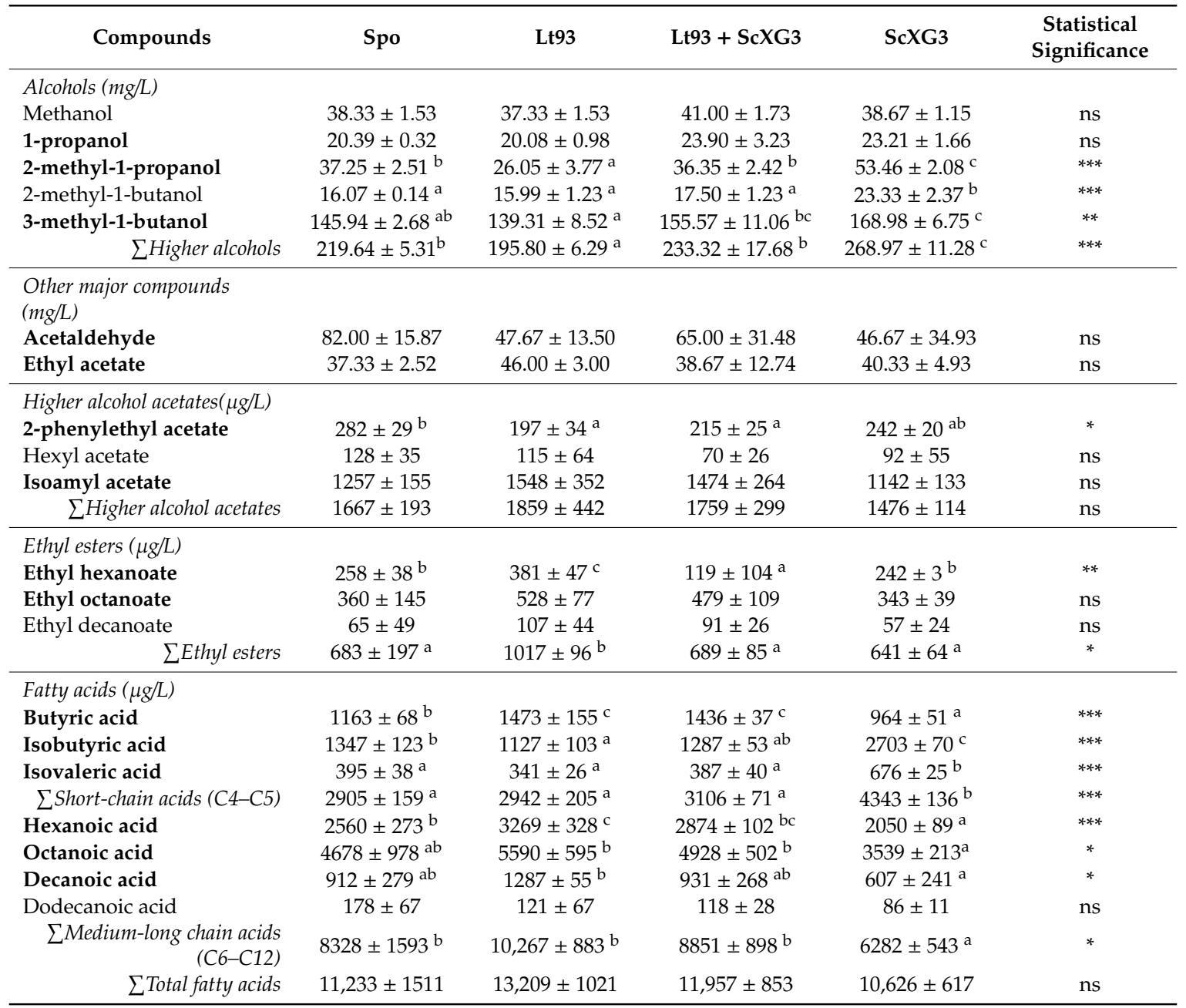

Data are mean values of three repetitions \pm Standard Deviation. Different letters $(a-c)$ in the same row indicate significant differences according to the Tukey's test $(p<0.05)$. The notations ${ }^{*}, * *$ and ${ }^{* * *}$ indicate significant differences among wines at $p<0.05, p<0.01$ and $p<0.001$, respectively. The ns notation indicates no significant differences at $p<0.05$ according to a one-way ANOVA. Those compounds of which the concentration is above their odor threshold in at least one of the trials are in bold.

\subsubsection{Mencía}

Table 4 summarizes the basic characteristics of Mencía wines fermented with different yeast strains. Significant differences were found for all parameters among the resulting wines. Alcohol content was lower in Lt93 wines (added as monoculture and in sequential fermentation) than in those fermented with S. cerevisiae. Sugar content was $<0.2 \mathrm{~g} / \mathrm{L}$ in S. cerevisiae fermentations (Sc71B and Spo), indicating the best fermentation performance of this species. A clear difference was also found in total acidity, which achieved values $>10 \mathrm{~g} / \mathrm{L}$ in Lt93 wines, mainly related to the higher values of lactic acid in these wines compared to S. cerevisiae ones. Consequently, $\mathrm{pH}$ of $\mathrm{Lt} 93$ wines was lower, despite the fact that the malic acid had been already consumed at the end of alcoholic fermentation. Volatile acidity was higher in Lt93 wines, but the values were below its threshold $(0.8 \mathrm{~g} / \mathrm{L})$. Wines from commercial strain Sc71B and spontaneous fermentation were similar in their basic characteristics, except for malic acid, glycerol and $\mathrm{SO}_{2}$ content. 
Table 4. Characteristics of Mencía wines elaborated with S. cerevisiae Sc71B, L. thermotolerans Lt93, sequential fermentation of both strains and by spontaneous fermentation (Spo).

\begin{tabular}{|c|c|c|c|c|}
\hline Chemical Parameter & Sc71B & Lt93 & Lt93+Sc71B & Spo \\
\hline Alcohol content (\%vol.) * & $12.5 \pm 0.1^{\mathrm{a}}$ & $11.7 \pm 0.0^{b}$ & $11.9 \pm 0.1^{b}$ & $12.3 \pm 0.1^{a}$ \\
\hline Glucose + fructose $(\mathrm{g} / \mathrm{L}) *$ & $0.2 \pm 0.1^{\mathrm{a}}$ & $3.6 \pm 0.3^{b}$ & $1.85 \pm 0.2^{\mathrm{c}}$ & $0.2 \pm 0.1^{\mathrm{a}}$ \\
\hline Total acidity (g tartaric acid /L)* & $5.3 \pm 0.1^{\mathrm{a}}$ & $10.1 \pm 0.0^{\mathrm{c}}$ & $10.3 \pm 0.0^{\mathrm{c}}$ & $6.8 \pm 0.0^{\mathrm{b}}$ \\
\hline Volatile acidity (g acetic acid/L) * & $0.24 \pm 0.06^{\mathrm{ab}}$ & $0.36 \pm 0.01 b c$ & $0.42 \pm 0.01^{\mathrm{c}}$ & $0.20 \pm 0.00^{\mathrm{a}}$ \\
\hline Lactic acid $(\mathrm{g} / \mathrm{L}) *$ & $0.2 \pm 0.1^{\mathrm{a}}$ & $7.1 \pm 0.1^{b}$ & $7.2 \pm 0.8^{b}$ & $0.4 \pm 0.1^{\mathrm{a}}$ \\
\hline Malic acid $(\mathrm{g} / \mathrm{L})^{*}$ & $1.4 \pm 0.1^{b}$ & $0.1 \pm 0.0^{\mathrm{a}}$ & $0.1 \pm 0.0^{\mathrm{a}}$ & $2.5 \pm 0.0^{\mathrm{c}}$ \\
\hline Tartaric acid $(\mathrm{g} / \mathrm{L})$ * & $2.6 \pm 0.0^{\mathrm{a}}$ & $3.2 \pm 0.0^{c}$ & $3.1 \pm 0.0^{b}$ & $2.6 \pm 0.0^{\mathrm{a}}$ \\
\hline $\mathrm{pH}(-)^{*}$ & $3.74 \pm 0.00^{\mathrm{a}}$ & $3.54 \pm 0.02^{b}$ & $3.57 \pm 0.00^{b}$ & $3.61 \pm 0.01^{b}$ \\
\hline Glycerol $(\mathrm{g} / \mathrm{L})$ * & $10 \pm 0.0^{\mathrm{a}}$ & $8.6 \pm 0.0^{c}$ & $9.1 \pm 0.0^{b}$ & $8.2 \pm 0.0^{\mathrm{d}}$ \\
\hline Total sulfur dioxide $(\mathrm{mg} / \mathrm{L})$ * & $27.5 \pm 0.7^{\mathrm{a}}$ & $40.0 \pm 0.7^{b}$ & $43.0 \pm 0.7^{b}$ & $38.5 \pm 0.7^{b}$ \\
\hline
\end{tabular}

Data are mean values of two replicate fermentations \pm SD. ${ }^{*}$ significant differences according to Tukey's test $(p<0.05)$. ${ }^{a-d}$ in the same row indicate significant differences among wines.

The results about aroma profile of Mencía wines indicated that 15 from a total of 18 identified volatile compounds showed significant differences among wines (Table 5). In addition, eight compounds, mainly acetates and ethyl esters, achieved concentrations above their odor threshold (Table S2). Wines fermented with S. cerevisiae showed a higher concentration of volatile compounds than Lt93 wines for all groups of compounds determined (Table 5), especially in esters and volatile organic acids. These results allowed a clear separation of wines by PCA (Figure 6) using those compounds with $\mathrm{OAV}>1$, alcohol content, total acidity and $\mathrm{pH}$. In this case, the first two components, PC1 and PC2, explained $95.4 \%$ of the variance. Lt93 and Lt93+Sc71B wines were plotted on the negative side of PC1 due to their low content of acetates and ethyl esters and their high total acidity. In contrast, wines made with S. cerevisiae Sc71B and by spontaneous fermentation were located in the positive side of PC1, characterized by higher concentrations of ethyl esters, acetates and alcohol than non-Saccharomyces wines. Sc71B wines appeared in the negative part of PC2 due to their higher content of acetates.

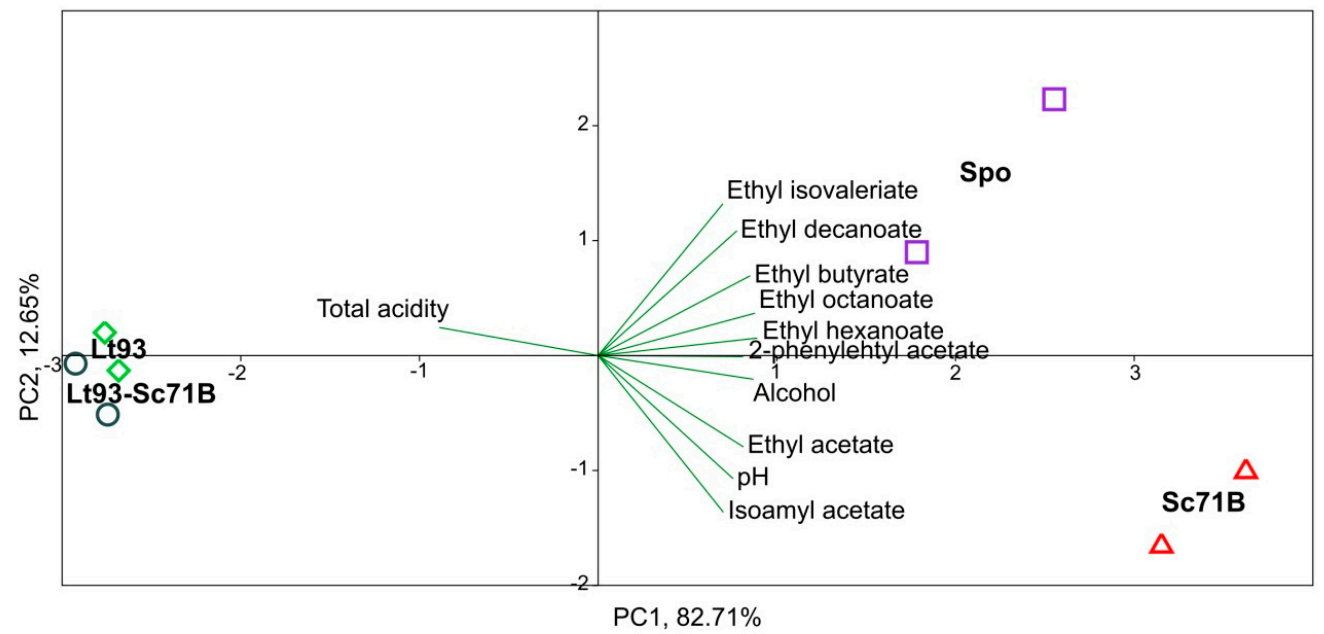

Figure 6. Principal component analysis of Mencía wines (PCA): biplot for the first two components (PC) for volatile compounds with OAV $>1$, alcohol content, total acidity and $\mathrm{pH}$. Wines were from fermentation with the following yeasts: Sc71B—S. cerevisiae Sc71B; Lt93—L. thermotolerans Lt93; Lt93-Sc71B - L. thermotolerans Lt93 and sequential inoculation of S. cerevisiae Sc71B; Spo: spontaneous fermentation. 
Table 5. Concentration of volatile compounds (expressed in $\mu \mathrm{g} / \mathrm{L}$ ) in Mencía wines elaborated with S. cerevisiae Sc71B, L. thermotolerans Lt93, sequential fermentation of both strains and by spontaneous fermentation (Spo).

\begin{tabular}{|c|c|c|c|c|c|}
\hline Compounds & Spo & Lt93 & Lt93+Sc71B & Sc71B & $\begin{array}{c}\text { Statistical } \\
\text { Significance }\end{array}$ \\
\hline \multicolumn{6}{|l|}{ Alcohols } \\
\hline Benzyl alcohol & $14 \pm 2$ & $8 \pm 0$ & $6 \pm 1$ & $14 \pm 4$ & ns \\
\hline 2-methyl-1-propanol & $1622 \pm 211^{\mathrm{a}}$ & $180 \pm 69^{c}$ & $1264 \pm 112^{a b}$ & $846 \pm 59^{b}$ & $* * *$ \\
\hline 3-methyl-1-butanol & $6350 \pm 163^{b}$ & $5311 \pm 198^{c}$ & $5081 \pm 185^{c}$ & $7155 \pm 61^{a}$ & $* * *$ \\
\hline 2-phenylethyl alcohol & $5604 \pm 360^{a b}$ & $3949 \pm 638^{b c}$ & $3266 \pm 349^{c}$ & $6491 \pm 159^{a}$ & $* *$ \\
\hline$\sum$ Higher alcohols & $13,577 \pm 733^{a}$ & $9440 \pm 370^{b}$ & $9612 \pm 53^{b}$ & $14,492 \pm 161^{a}$ & $* * *$ \\
\hline \multicolumn{6}{|l|}{ Ethyl esters } \\
\hline Ethyl butyrate & $150 \pm 20^{a}$ & $56 \pm 3^{b}$ & $55 \pm 5^{b}$ & $133 \pm 16^{a}$ & $* *$ \\
\hline Ethyl isovalerate & $20 \pm 1^{a}$ & $8 \pm 2^{c}$ & $9 \pm 1^{c}$ & $13 \pm 0^{b}$ & $* * *$ \\
\hline Ethyl hexanoate & $1148 \pm 153^{a}$ & $499 \pm 47^{b}$ & $476 \pm 37^{b}$ & $1258 \pm 20^{a}$ & $* * *$ \\
\hline Ethyl octanoate & $1405 \pm 124^{a}$ & $274 \pm 12^{b}$ & $229 \pm 31^{b}$ & $1360 \pm 69^{a}$ & $* * *$ \\
\hline Ethyl decanoate & $344 \pm 105^{a}$ & $86 \pm 6^{a b}$ & $41 \pm 16^{b}$ & $248 \pm 75^{a b}$ & $*$ \\
\hline Ethyl dodecanoate & $22 \pm 4$ & $12 \pm 9$ & $10 \pm 3$ & $27 \pm 7$ & ns \\
\hline$\sum$ Ethyl esters & $3090 \pm 407^{a}$ & $935 \pm 66^{b}$ & $819 \pm 52^{b}$ & $3040 \pm 49^{a}$ & $* * *$ \\
\hline Diethyl succinate & $943 \pm 46^{\mathrm{a}}$ & $84 \pm 26^{b}$ & $51 \pm 11^{b}$ & $1208 \pm 183^{a}$ & $* * *$ \\
\hline \multicolumn{6}{|l|}{ Acetates } \\
\hline Ethyl acetate & $7450 \pm 1972^{a b}$ & $3702 \pm 493^{b}$ & $4087 \pm 219^{b}$ & $10761 \pm 648^{\mathrm{a}}$ & $* *$ \\
\hline Isoamyl acetate & $1917 \pm 318^{b}$ & $1659 \pm 107^{b}$ & $1648 \pm 205^{b}$ & $2845 \pm 128^{\mathrm{a}}$ & * \\
\hline Hexyl acetate & $188 \pm 56$ & $143 \pm 2$ & $152 \pm 13$ & $216 \pm 47$ & ns \\
\hline 2-phenylehtyl acetate & $250 \pm 46^{\mathrm{ab}}$ & $185 \pm 17^{a b}$ & $123 \pm 26^{b}$ & $304 \pm 41^{a}$ & * \\
\hline EHigher alcohol acetates & $2355 \pm 327^{b}$ & $1987 \pm 91^{b}$ & $1923 \pm 192^{b}$ & $3365 \pm 134^{a}$ & ** \\
\hline \multicolumn{6}{|l|}{ Fatty acids (C6-C10) } \\
\hline Hexanoic acid & $132 \pm 25^{a}$ & $55 \pm 7^{b}$ & $41 \pm 9^{b}$ & $142 \pm 13^{a}$ & ** \\
\hline Octanoic acid & $244 \pm 42^{\mathrm{a}}$ & $32 \pm 7^{b}$ & $19 \pm 6^{b}$ & $242 \pm 20^{a}$ & $* * *$ \\
\hline \multirow{2}{*}{ Decanoic acid } & $49 \pm 2^{a}$ & $17 \pm 0^{b}$ & $10 \pm 1^{b}$ & $44 \pm 11^{\mathrm{a}}$ & $* *$ \\
\hline & $425 \pm 69^{a}$ & $105 \pm 14^{b}$ & $70 \pm 16^{b}$ & $428 \pm 44^{a}$ & $* * *$ \\
\hline
\end{tabular}

\footnotetext{
Data are mean values of two repetitions \pm Standard Deviation. Different letters $(\mathrm{a}-\mathrm{c})$ in the same row indicate significant differences according to the Tukey's test $(p<0.05)$. The notations ${ }^{*},{ }^{* *}$ and ${ }^{* * *}$ indicate significant differences between the different wines at $p<0.05, p<0.01$ and $p<0.001$, respectively. The ns notation indicates no significant differences at $p<0.05$ according to a one-way ANOVA. Those compounds which concentration is above their odor threshold in at least one of the trials are in bold.
}

\subsection{Sensory Evaluation of Treixadura Wines}

Chemical composition influences the sensory characteristics of wines [2]. Figure 7 represents the sensory profile of Treixadura wines obtained after fermentation with different yeast strains. Wines from Lt93 and spontaneous fermentations achieved higher scores than ScXG3 wines in most descriptors including global impression, aroma intensity and persistence with floral and fruity notes. The results are consistent with the presence of higher concentrations of esters in those wines. Regarding taste level, the differences were less noticeable being significant for structure and global quality in mouth. Lt93 and Spo wines stood out in all mouth descriptors as well as in global quality. As expected, Lt93 wines and those from spontaneous processes presented close profiles since the dynamics of yeast population during fermentation was similar. ScXG3 wines obtained the lowest scores in almost all descriptors. 


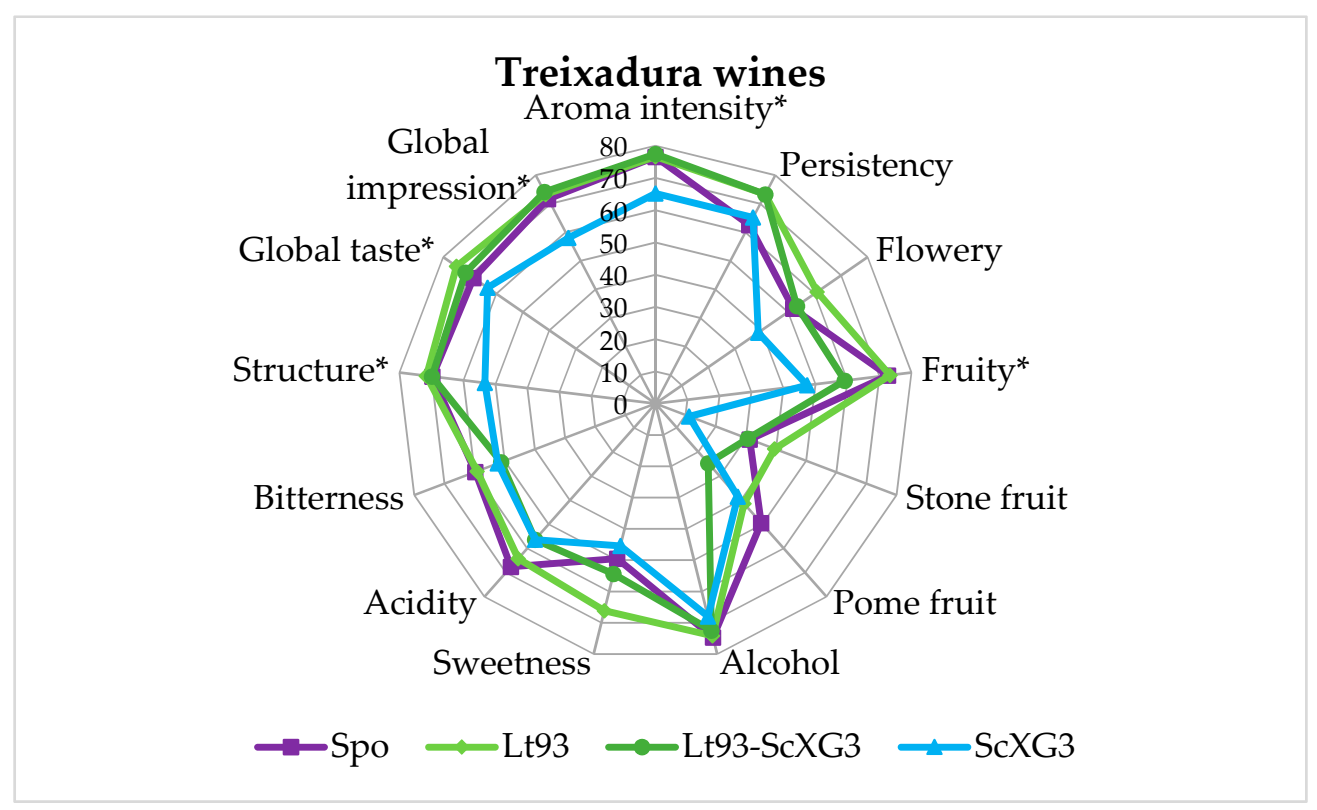

Figure 7. Sensory profile of Treixadura wines obtained with different yeast strains. ${ }^{*}$ Descriptor with significant differences among wines at $p<0.05$.

\section{Discussion}

Non-Saccharomyces yeasts have become an important tool in modern winemaking. Indeed, first considered detrimental, these yeast are nowadays recognized as desirable because they add complexity and regional signatures to wine $[6,7,27,28]$. Among non-Saccharomyces species, L. thermotolerans has been widely explored at industrial level because it increases the acidity of wines and influences their quality [9]. Numerous studies have reported that most of the positive contributions of L. thermotolerans to wines are strain-dependent; therefore, selection processes are considered to be necessary to increase the availability of strains. Moreover, it has been reported that $L$. thermotolerans is a quite good fermenter, but it requires the co-inoculation of a S. cerevisiae to complete fermentation $[6,10]$.

Previous studies carried out in EVEGA allowed the selection of Lt93, a strain of L. thermotolerans that increased lactic acid and total acidity and improved sensory properties of wine at laboratory scale [31]. Therefore, it was interesting to assess its oenological behavior with natural musts. In this work, L. thermotolerans Lt93 was used as monoculture and by sequential fermentation to ferment Treixadura and Mencía (traditional white and red cultivars in Galicia).

Kinetics of Treixadura fermentations showed a delay of L. thermotolerans Lt93 to begin fermentation; in fact, the fermentation curve of Lt93 and spontaneous process was similar (Figure 1) suggesting that Lt93 failed to compete with yeast population in the must. The analysis of yeast implantation at different stages of fermentation confirmed our supposition (Figure 2). The low frequency of Lt93 at the beginning of fermentation and its decrease during the process clearly indicated that it was unable to control fermentation. Instead, different strains of $S$. cerevisiae were responsible for fermentation as it happened in the spontaneous vinification. Sequential trials also follow this dynamic since they were already spontaneously dominated by ScR4, a wild strain of S. cerevisiae, when ScXG3 was added. We believe that although the initial amount of yeasts in Treixadura must was not particularly high, the proportion of S. cerevisiae (23\%), which is a better fermenter than L. thermotolerans, could have caused this outcome.

Regarding the amount of viable yeast, ScXG3 and Spo showed the conventional tendency to increase until the tumultuous phase and then decrease at the end of the fermentations; however, Lt 93 trials reached the maximum yeast counting at the beginning, but remained quite high until the end (Figure 1). A longer survival of S. cerevisiae in sequential fermentations has been described and explained by extra nutrients due to the death and autolysis of non-Saccharomyces [19]. 
The first studies on $L$. thermotolerans performance during fermentation had already reported a decrease in viability compared to S. cerevisiae [15]. Regarding mixed cultures, the permanence of L. thermotolerans depended on the timing of $S$. cerevisiae inoculation and the proportion of inocula. In co-cultures, $S$. cerevisiae strongly competed with L. thermotolerans, which completely died off after 7 days, but when the proportion of non-Saccharomyces in the inoculum increased, so did its permanence $[5,16]$. Despite the higher proportion of inoculum, L. thermotolerans Lt93 was unable to survive longer than 7 days in Treixadura fermentation (Ft in Figure 2) and to compete with wild S. cerevisiae population in the must. This lack of competitiveness in Treixadura trials was unexpected since L. thermotolerans had been often isolated from must and natural fermentations of this cultivar in DO Ribeiro and DO Monterrei in Galicia [30].

The loss of viability of non-Saccharomyces yeasts during fermentation has been related to several factors such as the presence of certain metabolites, redox conditions, the secretion of antimicrobial peptides by $S$. cerevisiae or cell-cell interactions [21,48-50]. In this case the presence of $\mathrm{SO}_{2}$, possible interactions with other yeast, and the high proportion of $S$. cerevisiae in the must could have been responsible for the failure of L. thermotolerans Lt93 in Treixadura fermentations.

In contrast, both strains inoculated into Mencía must, S. cerevisiae Sc71B and L. thermotolerans Lt93, begun fermentation within the first two days; although Lt93 slowed down its fermentation speed close to the end (Figure 3). As observed in Treixadura, when the S. cerevisiae strain (Sc71B in this case) was used as a single inoculum, it was the dominant strain during all the fermentation process (Figure 4). In Mencía, the non-Saccharomyces strain, Lt93, also carried out the fermentation successfully being the dominant yeast during all fermentation stages, except at the end. In the final stages, Lt 93 fermented in codominance with S. cerevisiae Sc71B (Figure 4). In sequential trials, Lt93 was replaced gradually by the second inoculum, $S$. cerevisiae Sc71B, as the fermentation progressed. This strain appeared also in codominance with ScXXII and other minor $S$. cerevisiae strains in spontaneous processes. The prevalence of $S$. cerevisiae Sc71B in the spontaneous fermentation indicated that this strain was well adapted to winery environment and Mencía must characteristics. The codominant strain, ScXXII, has also been described as resident yeast in EVEGA winery [51].

Available results about sequential fermentations indicated that the population of L. thermotolerans achieved higher counts and it also survived longer [11,21]. It is important to note that some of the previously mentioned studies were carried out using sterile/pasteurized musts. Fermentations with natural must confirmed that $L$. thermotolerans was dominant until 4-5 days, then decreased but survived until the 10th day [17]. Similar tendencies were observed in mixed cultures under co-inoculation [11]. In sequential trials $L$. thermotolerans was more competitive and remains at high concentration longer in some trials [11,24] or decreased fast after Saccharomyces inoculation [12,19]. Our results with Mencía agree with those findings and support the suitability of L. thermotolerans Lt93 for fermentation; in fact, it was the dominant yeast at the beginning and remained viable as a co-dominant with $S$. cerevisiae until the end.

The dynamic of yeast population during fermentation influences the chemical characteristics of wines. Accordingly, Treixadura and Mencía wines presented significant differences for certain parameters depending on the fermentative yeast strains, although the differences were less noticeable in Treixadura than in Mencía wines. Even so, significant differences were observed for parameters related to wine acidity despite the low implantation level of Lt93. In addition, Lt93 wines achieved higher values of total $\mathrm{SO}_{2}$ both in Treixadura and Mencía wines. The content of $\mathrm{SO}_{2}$ in L. thermotolerans wines has received little attention. It is known that the decrease in $\mathrm{pH}$ promotes better levels of molecular $\mathrm{SO}_{2}$ with low contents of total sulfites, making the fermentations and especially the ageing process safer [13]. Nisiotou et al. [12] reported higher total $\mathrm{SO}_{2}$ in wines involving L. thermotolerans compared to a commercial Saccharomyces as we found in this study; however, other authors [18] found lower concentrations of $\mathrm{SO}_{2}$ in sequential fermentations with $L$. thermotolerans.

A content of residual sugar $>2.0 \mathrm{~g} / \mathrm{L}$ in Treixadura wines indicated that fermentation was not complete, especially in ScXG3 wines. These results could be due to the lack of nutrients at the end 
of fermentation since this strain had shown to end fermentations that reached high alcohol content successfully [32,35]. Moreover, in the case of Lt93 fermentations, several S. cerevisiae strains were involved, but again, they were unable to complete fermentation.

The effects of L. thermotolerans Lt93 on the basic parameters of Mencía wines agree with those previously reported for this non-Saccharomyces yeast. The reduction of ethanol in Lt93 Mencía wines ranged from $0.4-0.8 \%$ vol. compared to $S$. cerevisiae fermentations. Similar values were reported in natural musts [19,22] and sterilized musts [20]; however, reductions of $1.0-1.5 \%$ were observed in industrial trials, although the presence residual sugars indicated that fermentations were not completed [11]. Other studies with mixed fermentations reported a lower ethanol reduction $(0.2 \%)[5,18]$ or did not find significant differences [21]. These findings confirmed that ethanol reduction is strain-dependent. Moreover, other factors such as the interaction among strains in mixed cultures could be involved. For instance, no differences were found for ethanol content in Treixadura wines, because $L$. thermotolerans was unable to control fermentation.

Among non-Saccharomyces yeasts, $L$. thermotolerans is particularly appreciated for its potential to enhance wine acidity. In this study Lt93 increased the total acidity of Mencía wines almost two-fold compared to $S$. cerevisiae related to a high production of lactic acid $(7.0 \mathrm{~g} / \mathrm{L})$. Similar values were found in pure fermentation of L. thermotolerans [15], although the maximum concentration of lactic acid exceeds $16 \mathrm{~g} / \mathrm{L}$ [52], and it was also found in L. thermotolerans Greek strains. Other studies evidenced a great variability among strains [22,25], such as in the range 1.8-12.0 g/L in Chardonnay wines [14]. In mixed fermentations, the production of lactic acid was also variable, for example from $0.22 \mathrm{~g} / \mathrm{L}$ [18], $0.53-4.42 \mathrm{~g} / \mathrm{L}$ [19], $3.18 \mathrm{~g} / \mathrm{L}$ [20] to $6.38 \mathrm{~g} / \mathrm{L}$ at winery scale [11]. The latter was similar to our results for Mencía. As a result of the lactic acid content, the total acidity of wines increased and the $\mathrm{pH}$ decreased. Natural or biological wine acidification is considered an interesting tool in winemaking, especially in those regions where wines reached high $\mathrm{pH}$ as a consequence of climate change from warmer climates/vintages [11,14,19-21]. In addition, acidity increase can have a positive impact on sensory properties and microbial stability of wine [6]. Despite the low survival of Lt93 in Treixadura fermentations, its wines had values of lactic acid and total acidity slightly higher than S. cerevisiae ones. Regarding malic acid degradation by L. thermotolerans, the available data also point to a great variability [9], but not completely, as found in this study with Mencía. A possible explanation could be a phenomenon of simultaneous malolactic and alcoholic fermentation.

The content of glycerol was lower in L. thermotolerans Lt93 monoculture and sequential wines than in S. cerevisiae and spontaneous fermentation with both cultivars (Mencía and Treixadura) regardless of Lt93 implantation (Tables 2 and 4). On the contrary, most studies reported that L. thermotolerans enhance glycerol concentration during sequential fermentations [11,16,19,20]. In addition, Hranilovic [14] has reported concentrations of glycerol between 3.9-8.0 g/L in pure L. thermotolerans fermentations. Glycerol contributes smoothness and complexity that influence wine quality positively.

Several studies have shown the potential of $L$. thermotolerans to modulate wine aroma $[9,25]$. Conversely, other authors did not find remarkable differences in wines fermented with this species at aroma level $[19,22,32]$. Higher alcohols are the largest group of volatile compounds in wine. Most studies reported that $L$. thermotolerans produced lower levels of higher alcohols than $S$. cerevisiae $[11,18,21,24,32]$. Mencía wines also followed this tendency mainly due to 3-methyl-1-butanol and 2-phenylethyl alcohol, although the concentrations were not above their odor threshold (Table 5). In contrast, for Treixadura wines, some of these compounds had OAVs > 1 (Table S1); ScXG3 wines presented the highest value of higher alcohols but none of the wines surpassed $300 \mathrm{mg} / \mathrm{L}$ (Table 3, Table S1), level below which they contribute to the aroma complexity [7]. However, in some cases, L. thermotolerans in mixed cultures yielded higher content of higher alcohols [5]. In particular, they found higher concentration of 2-phenyl ethanol, as reported by Gobbi et al. [11].

Esters and acetates are qualitatively important groups of volatile compounds because they impart desirable fruity and floral notes to wine [2]. Data about the production of esters by $L$. thermotolerans showed a great variability. In some cases, an increase in esters production was 
observed $[11,12,18,19,29]$, whereas other authors found a reduction in esters concentration with variations among strains [21,24]. In the current study, Mencía wines elaborated with Lt93 had lower concentrations of total ethyl esters, ethyl acetate and higher alcohols acetates than S. cerevisiae wines. The proportion of individual compounds differed between wines. Within ethyl esters, ethyl hexanoate achieved the highest concentration in Lt93 wines, whereas ethyl octanoate did so in S. cerevisiae wines. Regarding acetates of higher alcohol, the most abundant compound was isoamyl acetate in all Mencía wines. In addition, most esters presented an OAV $>1$ indicating their relevance at sensory level (Table S2). Aroma composition of Treixadura wines differed from Mencía. No significant difference was found for ethyl acetate and the higher alcohol acetates among Treixadura wines, except for 2-phenylethyl acetate content, which was higher in spontaneous fermentations (Table 3). The total concentration of ethyl esters was significantly higher in Lt93 monoculture wines mainly due to the content of ethyl hexanoate, although ethyl octanoate was the most abundant ethyl esters in these wines, as found in other studies [19]. These differences cannot be entirely attributed to L. thermotolerans since we have confirmed that its frequency was quite low throughout the fermentation. The combination of Lt93 and several S. cerevisiae strains were involved in this aroma profile. The results of Lt93 low rate of implantation during fermentation agreed with a previous study using sequential fermentation of these strains [32]; however, in that case, the concentration of esters and acetates was higher in pure ScXG3 wines than in sequential Lt93 wines.

The concentration of fatty acids in wines is relevant because they are precursors for the ethyl esters biosynthesis [53] and they are linked to unpleasant aromas. Indeed, fatty acids impart cheesy, sweaty, fatty and rancid notes to wines, but they can contribute to the aromatic equilibrium and complexity of wine $[54,55]$. It was found that concentrations of $4-10 \mathrm{mg} / \mathrm{L}$ of $\mathrm{C} 6-\mathrm{C} 10$ fatty acids provide a mild and pleasant aroma, whereas levels above $20 \mathrm{mg} / \mathrm{L}$ had a negative effect [56]. In Mencía and Treixadura assays, those wines with higher content of fatty acids also presented a higher concentration of ethyl esters. Regarding the influence of yeast, Lt93 Mencía wines showed lower concentrations of fatty acids than S. cerevisiae wines, although their content was below the odor threshold described for these volatiles OAV $<1$ (Table S2). The results agreed with those reported for pure cultures of L. thermotolerans [25] and in sequential fermentations [5,18,19,24]. In contrast, Treixadura wines had higher content of C6-C12 fatty acids when Lt93 was involved in fermentation. Furthermore, in these wines, the concentration of individual compounds was above its odor threshold (Table S1), and the sum of $\mathrm{C} 6-\mathrm{C} 10$ fatty acids was within the range of being positive to wine aroma.

Finally, the content of acetaldehyde presented an OAV $>1$ for all Treixadura wines; thus, it could influence their sensory properties. This compound is the main aldehyde formed during winemaking and its concentration is influenced by yeasts. In these wines, the content of acetaldehyde is lower than $100 \mathrm{mg} / \mathrm{L}$, a level that is considered to impart fruity flavors [55].

The results obtained in the current study confirmed that the use of L. thermotolerans Lt93 during the fermentation had an effect on population dynamics during fermentation. Variations in amount and proportion of yeast strains responsible for fermentation influenced the chemical composition of wines. Thus, the S. cerevisiae wines were clearly separated by PCA from wines from Lt93 (monoculture or sequentially fermented) based on their volatile composition.

In addition, these chemical differences had an influence at sensory level [2]. Benito [9] summarized the impact of $L$. thermotolerans on the sensory properties of wine as an increase in general acidity, overall impression, structure and aroma quality and a reduction in acetic acid. Our results with Treixadura wines confirmed these findings except for acidity; however, other author also found more intense notes of acidity in sequential fermentations with $L$. thermotolerans and $S$. cerevisiae $[11,12]$. The wines elaborated with Lt93 and by spontaneous fermentation were better scored than elaborated with ScXG3 ones for aroma intensity and floral and fruity notes, probably due to their higher content of esters. 


\section{Conclusions}

The results exposed in this study highlight the potential of L. thermotolerans Lt93. This non-Saccharomyces strain enhanced wine quality due to their acidifying properties, but also due to its impact on wine chemical composition. However, its competitiveness during natural fermentation was not always as satisfactory as expected. In Treixadura fermentations Lt93 failed to overgrow the indigenous yeast population in musts and could not start the fermentation. Even so, its presence and interaction with other yeasts during fermentation influenced positively the chemical and sensory properties of wine. Conversely, Lt93 was by far the most successful in dominating Mencía fermentations. Consequently, the acidity of Mencía Lt93 wines was almost two-fold higher than in S. cerevisiae wines. Therefore, further research is necessary to optimize the potential of this L. thermotolerans strain in Galician wine industry.

Future studies with red cultivars will need to focus on the proportion and timing of inoculum in sequential fermentations to find a protocol to take advantage of both species (L. thermotolerans and $S$. cerevisiae) benefits. Regarding white cultivars, the performance of $L$. thermotolerans will be evaluated using other ancestral white cultivars from Galicia.

Supplementary Materials: The following are available online at http://www.mdpi.com/2306-5710/6/2/36/s1, Table S1: Odor activity values (OAV) of volatile compounds determined in Treixadura wines made with different yeast strains; Table S2: Odor activity values (OAV) of volatile compounds determined in Mencía wines elaborated with different yeast strains.

Author Contributions: P.B. and D.C. conceived and designed the experiments; P.B., E.R., N.N. and D.C. performed the winemaking; E.R. and N.N. carried out the microbiological control of fermentations; D.C. carried out analytical determination; P.B. and D.C. analyzed the data; P.B. and D.C. wrote and critically revised the manuscript before submission. All authors have read and agreed to the published version of the manuscript.

Funding: This research received no external funding.

Acknowledgments: We would like to thank Cooperativa Cume do Avia for organic Treixadura must. David Castrillo thanks the INIA and European Social Fund for his FPI predoctoral contract.

Conflicts of Interest: The authors declare no conflict of interest.

\section{References}

1. Fleet, G.H.; Heard, G.M. Yeasts-Growth during fermentation. In Wine Microbiology and Biotechnology; Fleet, G.H., Ed.; Harwood Academic Publishers: Reading, UK, 1993; pp. $28-47$.

2. Swiegers, J.H.; Bartowsky, E.J.; Henschke, P.A.; Pretorius, I.S. Yeast and bacterial modulation of wine aroma and flavour. Aust. J. Grape Wine Res. 2005, 11, 139-173. [CrossRef]

3. Fleet, G.H. Wine yeasts for the future. FEMS Yeast Res. 2008, 8, 979-995. [CrossRef] [PubMed]

4. Jolly, N.P.; Augustyn, O.P.H.; Pretorius, I.S. The Role and Use of Non-Saccharomyces Yeasts in Wine Production. S. Afr. J. Enol. Vitic. 2006, 27, 15-39. [CrossRef]

5. Comitini, F.; Gobbi, M.; Domizio, P.; Romani, C.; Lencioni, L.; Mannazzu, I.; Ciani, M. Selected non-Saccharomyces wine yeasts in controlled multistarter fermentations with Saccharomyces cerevisiae. Food Microbiol. 2011, 28, 873-882. [CrossRef]

6. Jolly, N.P.; Varela, C.; Pretorius, I.S. Not your ordinary yeast: Non-Saccharomyces yeasts in wine production uncovered. FEMS Yeast Res. 2014, 14, 215-237. [CrossRef]

7. Padilla, B.; Gil, J.V.; Manzanares, P. Past and future of non-Saccharomyces yeasts: From spoilage microorganisms to biotechnological tools for improving wine aroma complexity. Front. Microbiol. 2016, 7, 87. [CrossRef]

8. Varela, C.; Dry, P.R.; Kutyna, D.R.; Francis, I.L.; Henschke, P.A.; Curtin, C.D.; Chambers, P.J. Strategies for reducing alcohol concentration in wine. Aust. J. Grape Wine Res. 2015, 21, 670-679. [CrossRef]

9. Benito, S. The impacts of Lachancea thermotolerans yeast strains on winemaking. Appl. Microbiol. Biotechnol. 2018, 102, 6775-6790. [CrossRef]

10. Ciani, M.; Comitini, F.; Mannazzu, I.; Domizio, P. Controlled mixed culture fermentation: A new perspective on the use of non-Saccharomyces yeasts in winemaking. FEMS Yeast Res. 2010, 10, 123-133. [CrossRef] 
11. Gobbi, M.; Comitini, F.; Domizio, P.; Romani, C.; Lencioni, L.; Mannazzu, I.; Ciani, M. Lachancea thermotolerans and Saccharomyces cerevisiae in simultaneous and sequential co-fermentation: A strategy to enhance acidity and improve the overall quality of wine. Food Microbiol. 2013, 33, 271-281. [CrossRef]

12. Nisiotou, A.; Mallouchos, A.; Tassou, C.; Banilas, G. Indigenous yeast interactions in dual-starter fermentations may improve the varietal expression of Moschofilero wine. Front. Microbiol. 2019, 10, 1712. [CrossRef] [PubMed]

13. Morata, A.; Loira, I.; Tesfaye, W.; Bañuelos, M.A.; González, C.; Suárez Lepe, J.A. Lachancea thermotolerans applications in wine technology. Fermentation 2018, 4, 53. [CrossRef]

14. Hranilovic, A.; Gambetta, J.M.; Schmidtke, L.; Boss, P.K.; Grbin, P.R.; Masneuf-Pomarede, I.; Bely, M.; Albertin, W.; Jiranek, V. Oenological traits of Lachancea thermotolerans show signs of domestication and allopatric differentiation. Sci. Rep. 2018, 8, 14312. [CrossRef] [PubMed]

15. Kapsopoulou, K.; Kapaklis, A.; Spyropoulos, H. Growth and fermentation characteristics of a strain of the wine yeast Kluyveromyces thermotolerans isolated in Greece. World J. Microbiol. Biotechnol. 2005, 21, 1599-1602. [CrossRef]

16. Kapsopoulou, K.; Mourtzini, A.; Anthoulas, M.; Nerantzis, E. Biological acidification during grape must fermentation using mixed cultures of Kluyveromyces thermotolerans and Saccharomyces cerevisiae. World J. Microbiol. Biotechnol. 2007, 23, 735-739. [CrossRef]

17. Mora, J.; Barbas, J.; Mulet, A. Growth of Yeast Species During the Fermentation of Musts Inoculated with Kluyveromyces thermotolerans and Saccharomyces cerevisiae. Am. J. Enol. Vitic. 1990, 41, 156-159.

18. Benito, S.; Hofmann, T.; Laier, M.; Lochbühler, B.; Schüttler, A.; Ebert, K.; Fritsch, S.; Röcker, J.; Rauhut, D. Effect on quality and composition of Riesling wines fermented by sequential inoculation with non-Saccharomyces and Saccharomyces cerevisiae. Eur. Food Res. Technol. 2015, 241, 707-717. [CrossRef]

19. Binati, R.L.; Lemos Junior, W.J.F.; Luzzini, G.; Slaghenaufi, D.; Ugliano, M.; Torriani, S. Contribution of non-Saccharomyces yeasts to wine volatile and sensory diversity: A study on Lachancea thermotolerans, Metschnikowia spp. and Starmerella bacillaris strains isolated in Italy. Int. J. Food Microbiol. 2020, 318, 108470. [CrossRef]

20. Benito, Á.; Calderón, F.; Palomero, F.; Benito, S. Quality and composition of airén wines fermented by sequential inoculation of Lachancea thermotolerans and Saccharomyces cerevisiae. Food Technol. Biotechnol. 2016, 54, 135-144. [CrossRef]

21. Balikci, E.K.; Tanguler, H.; Jolly, N.P.; Erten, H. Influence of Lachancea thermotolerans on cv. Emir wine fermentation. Yeast 2016, 33, 313-321. [CrossRef]

22. Binati, R.L.; Innocente, G.; Gatto, V.; Celebrin, A.; Polo, M.; Felis, G.E.; Torriani, S. Exploring the diversity of a collection of native non-Saccharomyces yeasts to develop co-starter cultures for winemaking. Food Res. Int. 2019, 122, 432-442. [CrossRef] [PubMed]

23. Vilela, A. Lachancea thermotolerans, the Non-Saccharomyces yeast that reduces the volatile acidity of wines. Fermentation 2018, 4, 56. [CrossRef]

24. Escribano-Viana, R.; González-Arenzana, L.; Portu, J.; Garijo, P.; López-Alfaro, I.; López, R.; Santamaría, P.; Gutiérrez, A.R. Wine aroma evolution throughout alcoholic fermentation sequentially inoculated with nonSaccharomyces/Saccharomyces yeasts. Food Res. Int. 2018, 112, 17-24. [CrossRef] [PubMed]

25. Escribano, R.; González-Arenzana, L.; Portu, J.; Garijo, P.; López-Alfaro, I.; López, R.; Santamaría, P.; Gutiérrez, A.R. Wine aromatic compound production and fermentative behaviour within different non-Saccharomyces species and clones. J. Appl. Microbiol. 2018, 124, 1521-1531. [CrossRef]

26. Bokulich, N.A.; Thorngate, J.H.; Richardson, P.M.; Mills, D.A. Microbial biogeography of wine grapes is conditioned by cultivar, vintage, and climate. Proc. Natl. Acad. Sci. USA 2014, 111, E139-E148. [CrossRef]

27. Bokulich, N.; Collins, T.; Masarweh, C.; Allen, G.; Heymann, H.; Ebeler, S.E.; Mills, D.A. Fermentation Behavior Suggest Microbial Contribution to Regional. MBio 2016, 7, 1-12. [CrossRef]

28. Knight, S.; Klaere, S.; Fedrizzi, B.; Goddard, M.R. Regional microbial signatures positively correlate with differential wine phenotypes: Evidence for a microbial aspect to terroir. Sci. Rep. 2015, 5, 1-10. [CrossRef]

29. Hranilovic, A.; Bely, M.; Masneuf-Pomarede, I.; Jiranek, V.; Albertin, W. The evolution of Lachancea thermotolerans is driven by geographical determination, anthropisation and flux between different ecosystems. PLoS ONE 2017, 12, e0184652. [CrossRef]

30. Castrillo, D.; Rabuñal, E.; Neira, N.; Blanco, P. Yeast diversity on grapes from Galicia, NW Spain: Biogeographical patterns and the influence of the farming system. Oeno ONE 2019, 53, 573-587. [CrossRef] 
31. Blanco, P.; Castrillo, D.; Graña, M.J.; Lorenzo, M.J.; Soto Vázquez, E. Evaluación de levaduras vínicas no convencionales para afrontar las consecuencias del cambio climático en bodega. Enoviticultura 2019, 58, $16-25$.

32. Castrillo, D.; Rabuñal, E.; Neira, N.; Blanco, P. Oenological potential of non-Saccharomyces yeasts to mitigate effects of climate change in winemaking: Impact on aroma and sensory profiles of Treixadura wines. FEMS Yeast Res. 2019, 19, 1-11. [CrossRef] [PubMed]

33. Bouzas-Cid, Y.; Falqué, E.; Orriols, I.; Mirás-Avalos, J.M. Effects of irrigation over three years on the amino acid composition of Treixadura (Vitis vinifera L.) musts and wines, and on the aromatic composition and sensory profiles of its wines. Food Chem. 2018, 240, 707-716. [CrossRef] [PubMed]

34. Bouzas-Cid, Y.; Trigo-Córdoba, E.; Orriols, I.; Falqué, E.; Mirás-Avalos, J. Influence of Soil Management on the Red Grapevine (Vitis vinifera L.) Mencía Must Amino Acid Composition and Wine Volatile and Sensory Profiles in a Humid Region. Beverages 2018, 4, 76. [CrossRef]

35. Blanco, P.; Mirás-Avalos, J.M.; Pereira, E.; Orriols, I. Fermentative aroma compounds and sensory profiles of Godello and Albariño wines as influenced by Saccharomyces cerevisiae yeast strains. J. Sci. Food Agric. 2013, 93, 2849-2857. [CrossRef] [PubMed]

36. Blanco, P.; Mirás-Avalos, J.M.; Suárez, V.; Orriols, I. Inoculation of Treixadura musts with autochthonous Saccharomyces cerevisiae strains: Fermentative performance and influence on the wine characteristics. Food Sci. Technol. Int. 2013, 19, 177-186. [CrossRef] [PubMed]

37. Pallmann, C.L.; Brown, J.A.; Olineka, T.L.; Cocolin, L.; Mills, D.A.; Bisson, L.F. Use of WL Medium to Profile Native Flora. Am. J. Enol. Vitic. 2001, 52, 198-203.

38. Esteve-Zarzoso, B.; Belloch, C.; Uruburu, F.; Querol, A. Identification of yeasts by RFLP analysis of the 5.8S rRNA gene and the two ribosomal internal transcribed spacers. Int. J. Syst. Bacteriol. 1999, 49, 329-337. [CrossRef]

39. Querol, A.; Barrio, E.; Huerta, T.; Ramon, D. Molecular monitoring of wine fermentations conducted by active dry yeast strains. Appl. Environ. Microbiol. 1992, 58, 2948-2953. [CrossRef]

40. International Organisation of Vine and Wine. Office International de la Vigne et du Vin Compendium of International Methods of Wine and Must Analysis; OIV: Paris, France, 2015.

41. Ferreira, V.; López, R.; Cacho, J.F. Quantitative determination of the odorants of young red wines from different grape varieties. J. Sci. Food Agric. 2000, 80, 1659-1667. [CrossRef]

42. Guth, H. Quantitation and Sensory Studies of Character Impact Odorants of Different White Wine Varieties. J. Agric. Food Chem. 1997, 45, 3027-3032. [CrossRef]

43. Etievant, P.X. Wine. In Volatile Compounds of Food and Beverages; Maarse, H., Ed.; Marcel Dekker: New York, NY, USA, 1991; pp. 483-546.

44. Francis, I.L.; Newton, J.L. Determining wine aroma from compositional data. Aust. J. Grape Wine Res. 2005, 11, 114-126. [CrossRef]

45. Vilanova, M.; Martínez, C. First study of determination of aromatic compounds of red wine from Vitis vinifera cv. Castañal grown in Galicia (NW Spain). Eur. Food Res. Technol. 2007, 224, 431-436. [CrossRef]

46. Englezos, V.; Rantsiou, K.; Cravero, F.; Torchio, F.; Pollon, M.; Fracassetti, D.; Ortiz-Julien, A.; Gerbi, V.; Rolle, L.; Cocolin, L. Volatile profile of white wines fermented with sequential inoculation of Starmerella bacillaris and Saccharomyces cerevisiae. Food Chem. 2018, 257, 350-360. [CrossRef] [PubMed]

47. Vilanova, M.; Escudero, A.; Graña, M.; Cacho, J. Volatile composition and sensory properties of North West Spain white wines. Food Res. Int. 2013, 54, 562-568. [CrossRef]

48. Nissen, P.; Nielsen, D.; Arneborg, N. Viable Saccharomyces cerevisiae cells at high concentrations cause early growth arrest of non-Saccharomyces yeasts in mixed cultures by a cell-cell contact-mediated mechanism. Yeast 2003, 20, 331-341. [CrossRef] [PubMed]

49. Ciani, M.; Comitini, F. Yeast interactions in multi-starter wine fermentation. Curr. Opin. Food Sci. 2015, 1, 1-6. [CrossRef]

50. Kemsawasd, V.; Branco, P.; Almeida, M.G.; Caldeira, J.; Albergaria, H.; Arneborg, N. Cell-to-cell contact and antimicrobial peptides play a combined role in the death of Lachanchea thermotolerans during mixed-culture alcoholic fermentation with Saccharomyces cerevisiae. FEMS Microbiol. Lett. 2015, 362. [CrossRef]

51. Blanco, P.; Mirás-Avalos, J.M.; Pereira, E.; Fornos, D.; Orriols, I. Modulation of chemical and sensory characteristics of red wine from Mencía by using indigenous Saccharomyces cerevisiae yeast strains. J. Int. Sci. Vigne Vin 2014, 48, 63-74. [CrossRef] 
52. Banilas, G.; Sgouros, G.; Nisiotou, A. Development of microsatellite markers for Lachancea thermotolerans typing and population structure of wine-associated isolates. Microbiol. Res. 2016, 193, 1-10. [CrossRef]

53. Saerens, S.M.G.; Delvaux, F.R.; Verstrepen, K.J.; Van Dijck, P.; Thevelein, J.M.; Delvaux, F.R. Parameters affecting ethyl ester production by Saccharomyces cerevisiae during fermentation. Appl. Environ. Microbiol. 2008, 74, 454-461. [CrossRef]

54. Callejon, R.M.; Clavijo, A.; Ortigueira, P.; Troncoso, A.M.; Paneque, P.; Morales, M.L. Volatile and sensory profile of organic red wines produced by different selected autochthonous and commercial Saccharomyces cerevisiae strains. Anal. Chim. Acta 2010, 660, 68-75. [CrossRef] [PubMed]

55. Gil, M.; Cabellos, J.M.; Arroyo, T.; Prodanov, M. Characterization of the volatile fraction of young wines from the Denomination of Origin “Vinos de Madrid" (Spain). Anal. Chim. Acta 2006, 563, 145-153. [CrossRef]

56. Shinohara, T. Gas Chromatographic Analysis of Volatile Fatty Acids in Wines. Agric. Biol. Chem. 1985, 49, 2211-2212.

(C) 2020 by the authors. Licensee MDPI, Basel, Switzerland. This article is an open access article distributed under the terms and conditions of the Creative Commons Attribution (CC BY) license (http://creativecommons.org/licenses/by/4.0/). 